\title{
Tricuspid valve dysplasia: A retrospective study of clinical features and outcome in dogs in the UK
}

\author{
Xavier Navarro-Cubas ${ }^{1, *}$, Valentina Palermo ${ }^{2}$, Anne French ${ }^{3}$, Sandra Sanchis-Mora ${ }^{4}$ and Geoff Culshaw ${ }^{5}$ \\ ${ }^{1}$ University of Liverpool, Small Animal Teaching Hospital, Leahurst Campus, Chester High Road, Neston, Wirral, \\ CH64 7TE, UK \\ ${ }^{2}$ Anderson and Moores Veterinary Specialists, The Granary, Bunstead Barns, Poles Lane, Hursley, Winchester, \\ Hampshire, SO21 2LL, UK \\ ${ }^{3}$ School of Veterinary Medicine, College of Medical, Veterinary and Life Sciences, University of Glasgow, Bearsden \\ Road, Bearsden, Glasgow, G61 1QH, UK \\ ${ }^{4}$ The Royal Veterinary College, Hawkshead Lane, North Mymms, Hatfield, Hertfordshire, AL9 7TA, UK \\ ${ }^{5} R(D) S V S$ Hospital for Small Animals, The University of Edinburgh, Easter Bush Veterinary Centre, Roslin, \\ Midlothian, EH25 9RG, UK
}

\begin{abstract}
The objective of this study was to determine the demographic, clinical and survival characteristics and to identify risk factors for mortality due to tricuspid valve dysplasia in UK dogs. Records of client-owned dogs diagnosed with tricuspid valve dysplasia at a referral centre were retrospectively reviewed. Only dogs diagnosed with tricuspid valve dysplasia based on the presence of a right-sided heart murmur identified prior to one year of age, and confirmed with Doppler echocardiography, were included. Dogs with concomitant cardiac diseases, pulmonary hypertension and/or trivial tricuspid regurgitation were excluded. Analysed data included signalment, reason for presentation, clinical signs, electrocardiographic and echocardiographic features, survival status and cause of death. Survival times and risk factors for mortality were evaluated using Kaplan-Meier curves and Cox regression. Eighteen dogs met inclusion criteria. Border collies were over-represented $(\mathrm{p}=0.014)$. Dogs were most frequently referred for investigation of heart murmur. The most common arrhythmia was atrial fibrillation $(n=3)$. Median survival time from diagnosis of tricuspid valve dysplasia was 2775 days (range 1-3696 days; $95 \%$ CI 1542.41-4007.59) and from onset of right-sided congestive heart failure was 181 days (range 1-2130 days; 95\% CI 0-455.59). Syncope was the sole risk factor for cardiac death. In this population of UK dogs, tricuspid valve dysplasia was uncommon but, when severe, frequently led to rightsided congestive heart failure. Prognosis was favourable for mild and moderate tricuspid dysplasia. Survival time was reduced with right-sided congestive heart failure but varied widely. Risk of cardiac death was significantly increased if syncope had occurred.

Keywords: Tricuspid valve dysplasia, Congestive heart failure, Atrial fibrillation, Survival time, Canine congenital heart disease.

\section{Introduction}

Tricuspid valve dysplasia (TVD) in dogs encompasses a spectrum of congenital malformations of the tricuspid valve (TV) apparatus including focal or diffuse thickening of the leaflets, underdevelopment or undifferentiated chordae tendineae (CT) and papillary muscles (PM), incomplete separation of valve components from the ventricular wall and focal agenesis of valvular tissue, resulting in valvular insufficiency or rarely stenosis (Famula et al., 2002; Andelfinger et al., 2003; MacDonald, 2006; Ohad et al., 2013; Adin, 2014; Lake-Bakaar et al., 2017) (Fig. $1)$.

In human cardiology, TVD is divided into two categories. "Ebstein's malformation (EM)" is characterised by the apical displacement of the basal

attachment of the valvular leaflets (septal, mural or both), and atrialisation of the right ventricle (RV). Confirmation requires objective echocardiographic or post-mortem measurements of the valvular displacement indexed to body weight (Shiina et al., 1984), cardiac catheterisation with an electrophysiology study (Choi et al., 2009) or cardiac MRI (Said et al., 2012). In "tricuspid valve dysplasia", the leaflets and tension apparatus are malformed but are not displaced (Becker et al., 1971; Lang et al., 1991). This terminology has been applied to veterinary cardiology (Eyster et al., 1977; Cave, 2001; Andelfinger et al., 2003; Takemura et al., 2003; Sousa et al., 2006; Choi et al., 2009). In dogs, TVD is a rare congenital disease, affecting most commonly Labrador retriever (Wright et al., 2001).
\end{abstract}


Overall prevalence has been reported as between 2 and $7.4 \%$ in shelter and specialist referral populations (Tidholm, 1997; Oliveira et al., 2011; Schrope, 2015). Heritability has been established in Labrador retrievers (Famula et al., 2002) and Dogues de Bordeaux (Ohad et al., 2013), and the disease has been mapped to chromosome 9 in Labrador retrievers (Andelfinger et al., 2003).

EM in dogs is even more rarely reported, with only isolated case reports in the literature (Eyster et al., 1977; Takemura et al., 2003; Sousa et al., 2006; Choi et al., 2009). None of the published studies on canine TVD have included UK dogs.

Because TVD applies to a range of abnormalities and severities, valvular insufficiency is variable, although severe disease can lead to right-sided congestive heart failure (R-CHF) in both young and adult dogs (Adin, 2014).

Prognostication remains challenging, since, to the authors' knowledge, risk factors for disease progression and mortality have not been determined.

The aims of this study were to use retrospective data to describe demographic, clinical and survival characteristics and to identify risk factors for mortality due to TVD in UK dogs.

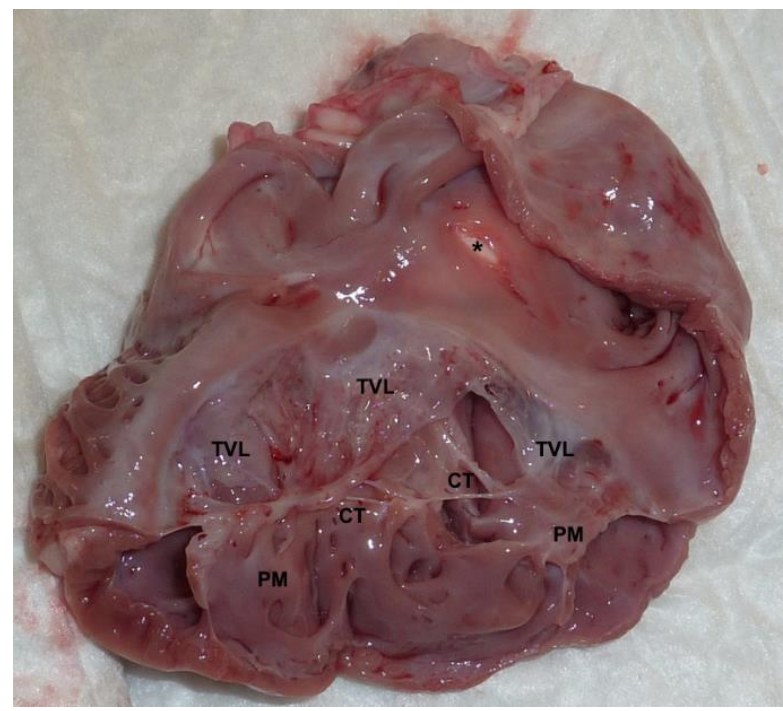

Fig. 1. Post-mortem image from a 3-month old Labrador retriever puppy diagnosed with TVD. There is diffuse marked thickening of the tricuspid leaflets (TVL), which exhibit irregular edges and are multifocally adhered to the endocardial surface. The chordae tendineae (CT) and papillary muscles (PM) are diffusely and severely shortened, fused and thickened. Marked right atrial enlargement is also observed. Please note the apparent defect $(*)$ in the interatrial septum; this was an iatrogenic cut accidentally done during preparation of the specimen (Image courtesy of Prof. Joanna Dukes-McEwan and Dr Sonja Fonfara).

\section{Materials and Methods}

Medical records of client-owned dogs diagnosed with TVD between 1998-2011 were retrospectively reviewed. Data including signalment, clinical history, physical examination, electrocardiographic (ECG) and echocardiographic findings, diagnosis, survival status and date and cause of death were collected from all cases. Inclusion criteria were diagnosis of TVD based on presence of a right-sided systolic murmur that had been first identified prior to one year of age, and a comprehensive Doppler echocardiographic study. Exclusion criteria were incomplete case record and/or echocardiographic study, presence of trivial tricuspid regurgitation (TR) and/or pulmonary hypertension (TR vmax. $>2.8 \mathrm{~m} / \mathrm{s}$ ) (Adams et al., 2017). Dogs with concomitant cardiac diseases were included in order to identify conditions associated with TVD, and breed and sex predispositions, but, otherwise excluded from all other statistical analysis. Where collapse had been reported, and no non-cardiac cause had been identified on investigation, it was assumed to be cardiac syncope. Heart murmurs (HM) were categorised according to location and intensity (Grades I to VI). Standard sixlead electrocardiograms were reviewed.

Echocardiograms were performed on all dogs, without sedation, by an ECVIM-CA or RCVS-diplomate, or by a diplomate-supervised ECVIM-CA-enrolled resident. Measurements were made offline by the same echocardiographer. Echocardiographic findings consistent with a diagnosis of TVD, including valvular abnormalities, are listed in Table 1 (Boon, 2011a; Ohad et al., 2013; Adin, 2014; Schrope, 2015; Bussadori and Pradelli, 2016) (Fig. 2). Tethering of the septal leaflet was only confirmed if the leaflet was attached to the septal wall by a short CT that was subjectively preventing its closure (Boon, 2011b) (Fig. 2).

Colour-flow Doppler was used to confirm TR, which was semi-quantitatively assessed by comparing jet area with right atrial size (Table 1). Trivial TR was not an inclusion criterion, as it is very common in healthy dogs (Boon, 2011c; Ohad et al., 2013; Bussadori and Pradelli, 2016). Tricuspid stenosis was defined as the presence of diastolic doming or reduced diastolic excursion of the TV leaflets, reduced TV orifice diameter, increased diastolic pressure gradient between right atrium (RA) and $\mathrm{RV}(>3 \mathrm{mmHg}$ ), and/or presence of tricuspid inflow colour variance with Nyquist limit set at 0.7-1.0 m/s (Lake-Bakaar et al., 2017).

Right-sided volume overload was subjectively assessed based on the right atrial and right ventricular size, viewed from the right parasternal long axis four chamber view, and by comparing chamber proportions with left atrial and left ventricular size (Lang et al., 2006) as indicated in Table 1. 
Table 1. Summary of the echocardiographic abnormalities associated with tricuspid valve dysplasia.

\begin{tabular}{|c|c|c|c|}
\hline \multirow{7}{*}{$\begin{array}{l}\text { Tricuspid valve } \\
\text { apparatus - } \\
\text { morphological } \\
\text { abnormalities }\end{array}$} & \multirow{5}{*}{ Valve leaflets } & \multicolumn{2}{|c|}{ Dysplastic leaflets may be thickened, clubbed, shortened, elongated or fused. } \\
\hline & & \multicolumn{2}{|c|}{ Valvular leaflet elongation: more commonly observed in the parietal leaflet } \\
\hline & & \multirow{2}{*}{ Valvular tethering } & $\begin{array}{l}\text { Septal leaflet subjectively attached to the septal wall by } \\
\text { short CT, preventing its normal closure. }\end{array}$ \\
\hline & & & Can lead to non-coaptation of the valvular leaflets \\
\hline & & Tricuspid stenosis & $\begin{array}{l}\text { Considered if presence of diastolic doming or reduced } \\
\text { excursion of the TV leaflets, reduced TV orifice diameter, } \\
\text { increased diastolic pressure gradient between RA and RV ( }>3 \\
\mathrm{mmHg} \text { ), and/or presence of tricuspid inflow colour variance } \\
\text { with Nyquist limit set at } 0.7-1.0 \mathrm{~m} / \mathrm{s}\end{array}$ \\
\hline & Chordae tendineae & \multicolumn{2}{|c|}{ Dysplastic chordae include thickened, shortened or even absent chordae } \\
\hline & Papillary muscles & \multicolumn{2}{|c|}{$\begin{array}{l}\text { Dysplastic papillary muscles include shortened, elongated, fused or with direct } \\
\text { attachment to the valve leaflets }\end{array}$} \\
\hline \multirow{5}{*}{$\begin{array}{l}\text { Tricuspid } \\
\text { regurgitation }\end{array}$} & \multicolumn{3}{|c|}{ Confirmed by Colour-flow and spectral Doppler echocardiography } \\
\hline & \multirow{3}{*}{\multicolumn{2}{|c|}{$\begin{array}{l}\text { Severity semi-quantitatively assessed by } \\
\text { colour flow: TR jet size subjectively } \\
\text { compared with right atrial size (left apical } \\
\text { 4-chamber view optimised for RA \& RV) }\end{array}$}} & $<$ quarter of the RA area \\
\hline & & & Quarter to half of the RA area \\
\hline & & & Over half of the RA area \\
\hline & \multicolumn{3}{|c|}{$\begin{array}{l}\text { Trivial TR: Small TR jet with narrow jet origin, extending only a short distance from the valve leaflets. } \\
\text { Timing is brief, with TR early in systole. }\end{array}$} \\
\hline \multirow{6}{*}{$\begin{array}{l}\text { Right-sided } \\
\text { volume overload } \\
\text { (assessed from } \\
\text { right parasternal } \\
\text { long axis } 4 \\
\text { chamber view, } \\
\text { tipped to } \\
\text { optimise RA \& } \\
\text { RV) }\end{array}$} & \multirow{3}{*}{ RA enlargement } & Mild & RA subjectively remains smaller than LA \\
\hline & & Moderate & RA diameter subjectively is of similar size to the LA diameter \\
\hline & & Severe & RA subjectively has larger diameter than LA \\
\hline & \multirow{3}{*}{$\begin{array}{l}\text { RV enlargement } \\
\text { (normally RV > } \\
\text { one third LV } \\
\text { size) }\end{array}$} & Mild & RV subjectively remains smaller than LV \\
\hline & & Moderate & RV subjectively has similar size to the LV \\
\hline & & Severe & $\mathrm{RV}$ subjectively is larger than the LV \\
\hline
\end{tabular}

Severity of TVD was subjectively graded as mild, moderate or severe according to echocardiographic appearance of the TV apparatus, severity of right-sided volume overload and TR jet size (Zoghbi et al., 2003). Cases compatible with EM were not categorised separately from the TVD population. This was because only subjective assessment of apical displacement of the valvular apparatus was available, and not objective assessment (indexing to body weight) (Kornreich and Moïse, 1997), cardiac catheterisation with an electrophysiology study (Choi et al., 2009) or postmortem confirmation (Kornreich and Moïse, 1997) were available.
Statistical analysis used SPSS Statistics (IBM 2013) and Microsoft Excel.

Demographic variables were explored and described using proportions for categorical data and median, range and $95 \%$ confidence interval (CI) for continuous data. For continuous data, normality of distribution was verified by Kolmogorov-Smirnov's test and visual assessment of Q-Q plots and histograms. Breed overrepresentation was calculated using Chi-square analysis, comparing the proportion of a breed between the TVD population and the entire hospital population. For this analysis, we included dogs with TVD and concomitant cardiac diseases. 

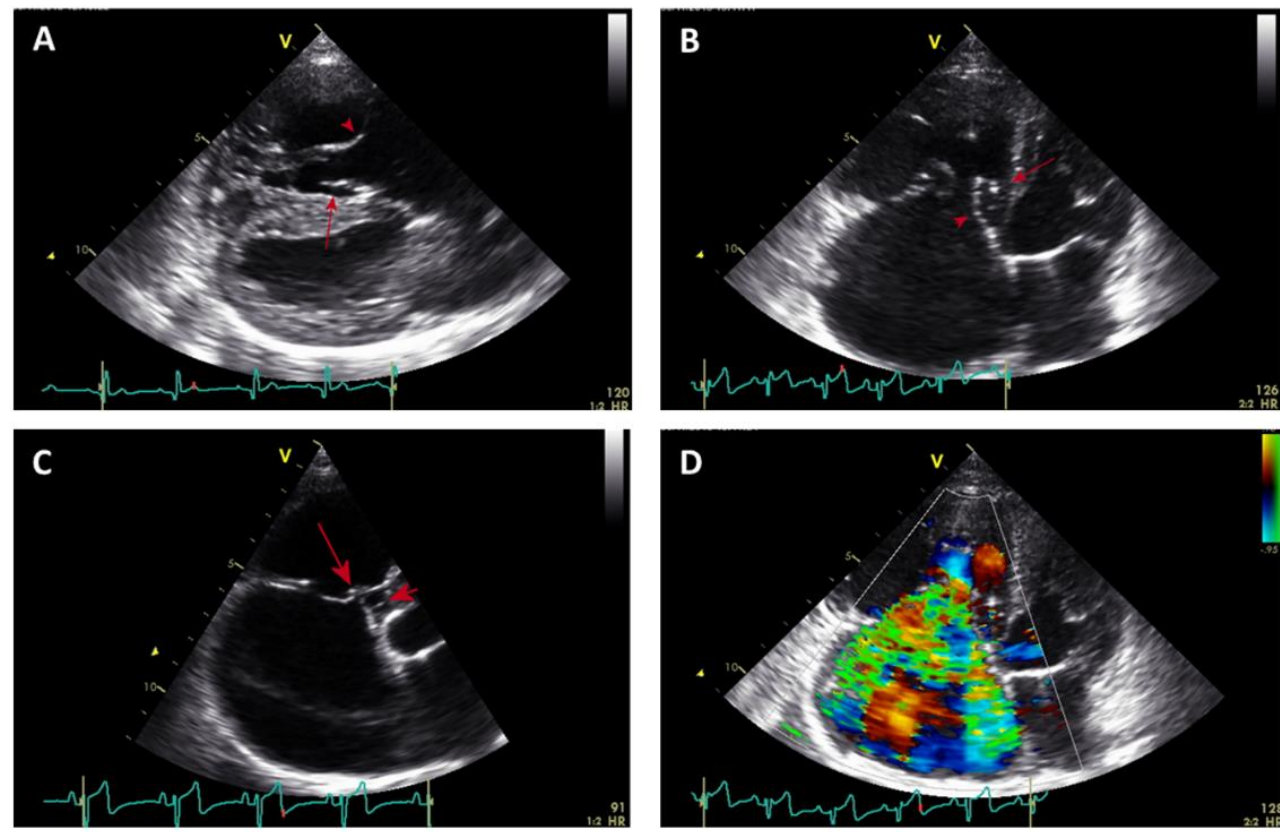

Fig. 2. (A): Right parasternal four-chamber long axis view showing right atrial and right ventricular dilation. The papillary muscle subjectively appears directly attached to the mural tricuspid valve leaflet (arrow head). The septal leaflet subjectively appears tethered to the septal wall (arrow) causing the incomplete occlusion of the tricuspid orifice in systole. (B): Left apical four-chamber view, optimised for the right atrium and right ventricle, showing severe right atrial and right ventricular dilation. The mural leaflet of the tricuspid valve subjectively appears markedly elongated. The septal leaflet appears tethered to the septal wall by short chordae tendineae (arrow), with the middle portion of the leaflet buckling away from the septum (arrow head). (C): Left apical four-chamber view, optimised for the right atrium and right ventricle, showing severe right atrial dilation. The mural leaflet of the tricuspid valve is markedly elongated, and is responsible for the occlusion of almost all the tricuspid valve orifice. The septal leaflet subjectively appears tethered to the septal wall by short chordae tendineae (arrow head), and the middle portion bows away from the interventricular septum. (D): Colour Doppler left apical four-chamber view, optimised for the right atrium and right ventricle, showing severe tricuspid regurgitation. Colour variance occupies the complete area of the right atrium.

The relationships between TVD severity, presence of an arrhythmia and heart murmur intensity were evaluated using a Fisher's exact test. The Kaplan-Meier method was used to estimate median survival times (MST) and plot time to event curves. The survival time (ST) study included two study periods: ST from diagnosis of TVD, and from diagnosis of R-CHF. The end-point for survival analysis was cardiac death, defined as euthanasia or natural death due to sudden death or worsening of R-CHF as a result of TVD. A questionnaire and telephone interview with referring veterinarians or the owners determined the status of each animal.

Dogs lost to follow-up (LTFU) were included in the survival analysis, left censored at the time of the last visit. Non-cardiac deaths and dogs still alive were also censored. Univariate analysis, using Cox regression, of the following risk factors was performed to determine whether there was any relationship with time to death from TVD diagnosis: age, sex, breed, TVD severity, HM intensity, R-CHF (at any point), syncope and arrhythmia. Multivariate analysis was not performed due to the low event rate. Significance of all tests was set at $\mathrm{p}<0.05$.

\section{Results}

During the study period, a total of $29 \mathrm{dogs}$ were identified with TVD, which was $1.1 \%$ of the population referred for cardiology evaluation. The two most common breeds with TVD were Labrador retrievers $(\mathrm{n}=7,24 \%)$ and Border collies $(\mathrm{n}=5,17 \%)$. Compared with the overall hospital population, only Border collies were over-represented $(\mathrm{p}=0.015)$ (Labradors $\mathrm{p}=0.219)$. Both sexes were diagnosed with the condition (female $\mathrm{n}=17$, 59\%; male $\mathrm{n}=12,41 \%)$. TVD was the sole cardiac disease in 18 dogs (62\% of all dogs with TVD, Table 2). In the remaining $11 \mathrm{dogs}(38 \%)$, concomitant cardiac diseases were diagnosed including mitral valve dysplasia $(n=4 ; 14 \%)$, pulmonic stenosis $(n=3 ; 10 \%)$, atrial septal defect $(n=2)$, and subaortic stenosis $(n=1)$ and left-to-right patent ductus arteriosus $(n=1)$ (Table 3). Dogs with isolated TVD were referred for investigation of a HM $(n=14,78 \%)$, syncope $(n=3$, $17 \%)$, presence of an arrhythmia $(n=1)$, signs consistent with R-CHF $(n=1)$, non-cardiac collapse $(n=1)$ and vomiting $(n=1)$ (Table 2). Age at diagnosis of TVD ranged between 50 days and 13 years (median $=252.5$ days). Twelve dogs (67\%) were less than one-year-old at time of diagnosis (Table 2). 
Table 2. Demographic information of dogs diagnosed with isolated TVD.

\begin{tabular}{|c|c|c|c|c|c|c|c|c|c|c|}
\hline Dog & Breed & $\begin{array}{l}\text { Reason for } \\
\text { presentation }\end{array}$ & Heart murmur & Diagnosis & $\begin{array}{c}\text { Age at TVD } \\
\text { diagnosis } \\
\text { (days) }\end{array}$ & $\mathrm{RCHF}$ & $\begin{array}{c}\text { Age at RCHF } \\
\text { diagnosis } \\
\text { (days) }\end{array}$ & $\begin{array}{c}\text { Signs of } \\
\text { CHF }\end{array}$ & $\begin{array}{l}\text { Status at the } \\
\text { end of the } \\
\text { study }\end{array}$ & $\begin{array}{l}\text { Age at } \\
\text { death } \\
\text { (days) }\end{array}$ \\
\hline $\begin{array}{c}\text { Dog } \\
1\end{array}$ & Border collie & $\begin{array}{l}\text { Heart } \\
\text { murmur }\end{array}$ & $\begin{array}{c}\text { IV/VI } \\
\text { pansystolic } \\
\text { right apical }\end{array}$ & Severe TVD & 80 & Yes & 2713 & Ascites & $\begin{array}{c}\text { Euthanasia. } \\
\text { Unresponsive } \\
\text { RCHF }\end{array}$ & 2855 \\
\hline $\begin{array}{c}\text { Dog } \\
2\end{array}$ & Boxer & $\begin{array}{c}\text { Non-Cardiac } \\
\text { collapse }\end{array}$ & $\begin{array}{l}\text { II/VI systolic } \\
\text { right basilar }\end{array}$ & $\begin{array}{l}\text { Moderate } \\
\text { TVD }\end{array}$ & 216 & No & & - & $\begin{array}{l}\text { Euthanasia. } \\
\text { Spleen } \\
\text { rupture } \\
\text { haemoabdom } \\
\text { en. }\end{array}$ & 3912 \\
\hline $\begin{array}{c}\text { Dog } \\
3\end{array}$ & Doberman & $\begin{array}{l}\text { Heart } \\
\text { murmur }\end{array}$ & $\begin{array}{l}\text { III/VI systolic } \\
\text { right apical }\end{array}$ & Mild TVD & 2535 & No & & - & $\begin{array}{l}\text { Euthanasia. } \\
\text { Lymphoma }\end{array}$ & 4781 \\
\hline $\begin{array}{c}\text { Dog } \\
4\end{array}$ & Border collie & $\begin{array}{c}\text { Heart } \\
\text { murmur, } \\
\text { ascites, } \\
\text { syncope, } \\
\text { pleural } \\
\text { effusion }\end{array}$ & $\begin{array}{c}\text { V/VI } \\
\text { pansystolic } \\
\text { apical } \\
\text { bilaterally }\end{array}$ & Severe TVD & 318 & Yes & 322 & $\begin{array}{l}\text { Ascites, } \\
\text { pleural } \\
\text { effusion } \\
\text { (mild), } \\
\text { jugular } \\
\text { distensio } \\
\text { n/pulse }\end{array}$ & $\begin{array}{c}\text { Euthanasia. } \\
\text { Unresponsive } \\
\text { RCHF }\end{array}$ & 2297 \\
\hline $\begin{array}{c}\text { Dog } \\
5\end{array}$ & Border collie & $\begin{array}{c}\text { Heart } \\
\text { murmur }\end{array}$ & $\begin{array}{l}\text { V/VI } \\
\text { pansystolic } \\
\text { right apical }\end{array}$ & Severe TVD & 2588 & No & & - & $\begin{array}{l}\text { Natural death. } \\
\text { Immune- } \\
\text { mediated } \\
\text { neutropenia. }\end{array}$ & 5040 \\
\hline $\begin{array}{l}\operatorname{Dog} \\
6\end{array}$ & Pointer & $\begin{array}{c}\text { Heart } \\
\text { murmur }\end{array}$ & $\begin{array}{l}\text { IV/VI systolic } \\
\text { right basilar }\end{array}$ & Severe TVD & 50 & LTFU & LTFU & - & $\begin{array}{c}\text { Lost to follow } \\
\text { up }\end{array}$ & 50 \\
\hline $\begin{array}{c}\text { Dog } \\
7\end{array}$ & $\begin{array}{l}\text { Labrador } \\
\text { retriever }\end{array}$ & $\begin{array}{l}\text { Heart } \\
\text { murmur }\end{array}$ & $\begin{array}{c}\text { IV/VI } \\
\text { pansystolic } \\
\text { right apical }\end{array}$ & Severe TVD & 62 & No & & - & Alive & 892 \\
\hline $\begin{array}{c}\text { Dog } \\
8\end{array}$ & $\begin{array}{l}\text { Golden } \\
\text { retriever }\end{array}$ & $\begin{array}{c}\text { Heart } \\
\text { murmur }\end{array}$ & $\begin{array}{c}\text { III/VI } \\
\text { pansystolic } \\
\text { right apical }\end{array}$ & $\begin{array}{l}\text { Moderate } \\
\text { TVD }\end{array}$ & 1481 & No & & - & $\begin{array}{l}\text { Euthanasia. } \\
\text { Renal failure }\end{array}$ & 2178 \\
\hline $\begin{array}{c}\text { Dog } \\
9\end{array}$ & $\begin{array}{r}\text { German } \\
\text { shepherd }\end{array}$ & $\begin{array}{c}\text { Heart } \\
\text { murmur }\end{array}$ & $\begin{array}{c}\text { V/VI } \\
\text { pansystolic } \\
\text { right apical }\end{array}$ & Severe TVD & 199 & Yes & 2829 & Ascites & $\begin{array}{c}\text { Euthanasia. } \\
\text { Unresponsive } \\
\text { RCHF }\end{array}$ & 3644 \\
\hline $\begin{array}{c}\text { Dog } \\
10\end{array}$ & $\begin{array}{c}\text { Golden } \\
\text { retriever }\end{array}$ & $\begin{array}{c}\text { Heart } \\
\text { murmur }\end{array}$ & $\begin{array}{c}\text { III/VI } \\
\text { holosystolic } \\
\text { right apical }\end{array}$ & Mild TVD & 4745 & No & & - & $\begin{array}{c}\text { Euthanasia. } \\
\text { Hind limb } \\
\text { weakness. } \\
\text { Lumbosacral } \\
\text { pain. }\end{array}$ & 4988 \\
\hline $\begin{array}{c}\text { Dog } \\
11\end{array}$ & $\begin{array}{l}\text { Labrador } \\
\text { retriever }\end{array}$ & Vomiting & $\begin{array}{c}\text { II/VI } \\
\text { holosystolic } \\
\text { right apical }\end{array}$ & Mild TVD & 858 & No & & - & Alive & 2155 \\
\hline $\begin{array}{c}\text { Dog } \\
12\end{array}$ & $\begin{array}{c}\text { Golden } \\
\text { retriever }\end{array}$ & $\begin{array}{c}\text { Heart } \\
\text { murmur }\end{array}$ & $\begin{array}{l}\text { I/VI systolic } \\
\text { right apical }\end{array}$ & Mild TVD & 205 & No & & - & Alive & 1393 \\
\hline $\begin{array}{l}\text { Dog } \\
13\end{array}$ & $\begin{array}{l}\text { Springer } \\
\text { spaniel }\end{array}$ & $\begin{array}{l}\text { Heart } \\
\text { murmur }\end{array}$ & $\begin{array}{l}\text { V/VI } \\
\text { pansystolic } \\
\text { right basilar }\end{array}$ & Severe TVD & 109 & Yes & 749 & Ascites & $\begin{array}{c}\text { Euthanasia. } \\
\text { Unresponsive } \\
\text { RCHF }\end{array}$ & 773 \\
\hline $\begin{array}{c}\text { Dog } \\
14\end{array}$ & Border collie & $\begin{array}{c}\text { Heart } \\
\text { murmur, } \\
\text { syncope }\end{array}$ & $\begin{array}{c}\text { VI/VI } \\
\text { pansystolic } \\
\text { apical } \\
\text { bilaterally }\end{array}$ & Severe TVD & 60 & Yes & 61 & Ascites & $\begin{array}{c}\text { Euthanasia. } \\
\text { Unresponsive } \\
\text { RCHF }\end{array}$ & 60 \\
\hline $\begin{array}{c}\text { Dog } \\
15\end{array}$ & Bullmastiff & Arrhythmia & $\begin{array}{c}\text { II/VI } \\
\text { holosystolic } \\
\text { right apical }\end{array}$ & Severe TVD & 356 & Yes & 361 & $\begin{array}{l}\text { Ascites } \\
\text { and } \\
\text { pleural } \\
\text { effusion }\end{array}$ & $\begin{array}{c}\text { Natural death. } \\
\text { Cardiorespirat } \\
\text { ory arrest }\end{array}$ & 2486 \\
\hline $\begin{array}{l}\text { Dog } \\
16\end{array}$ & $\begin{array}{l}\text { Labrador } \\
\text { retriever }\end{array}$ & Syncope & $\begin{array}{l}\text { III/VI systolic } \\
\text { right apical }\end{array}$ & Severe TVD & 289 & Yes & 293 & Ascites & $\begin{array}{c}\text { Euthanasia. } \\
\text { Unresponsive } \\
\text { RCHF }\end{array}$ & 363 \\
\hline $\begin{array}{c}\text { Dog } \\
17\end{array}$ & Cross breed & $\begin{array}{l}\text { Heart } \\
\text { murmur }\end{array}$ & $\begin{array}{c}\text { IV/VI } \\
\text { pansystolic } \\
\text { right basilar }\end{array}$ & Severe TVD & 81 & LTFU & LTFU & & LTFU & 841 \\
\hline $\begin{array}{c}\text { Dog } \\
18\end{array}$ & $\begin{array}{l}\text { Labrador } \\
\text { retriever }\end{array}$ & $\begin{array}{c}\text { Heart } \\
\text { murmur }\end{array}$ & $\begin{array}{c}\text { IV/VI systolic } \\
\text { right apical }\end{array}$ & Mild TVD & 2166 & No & & & Alive & 2761 \\
\hline
\end{tabular}


Table 3. Demographic information of dogs diagnosed with TVD and other concomitant cardiac diseases*.

\begin{tabular}{|c|c|c|c|c|c|}
\hline Dog & Breed & $\begin{array}{l}\text { Reason for } \\
\text { presentation }\end{array}$ & Heart murmur & Diagnosis & Status at the end of the study \\
\hline Dog 19 & $\begin{array}{l}\text { Labrador } \\
\text { retriever }\end{array}$ & Heart murmur & $\begin{array}{l}\text { II/VI holosystolic } \\
\text { left basilar }\end{array}$ & $\begin{array}{c}\text { Mild TVD + mild } \\
\text { SAS }\end{array}$ & Alive \\
\hline Dog 20 & $\begin{array}{l}\text { Springer } \\
\text { spaniel }\end{array}$ & Heart murmur & $\begin{array}{c}\text { IV/VI systolic left } \\
\text { apical }\end{array}$ & $\begin{array}{c}\text { Mild TVD + Mild } \\
\text { MVD }\end{array}$ & Lost to follow up \\
\hline $\operatorname{Dog} 21$ & $\begin{array}{l}\text { Labrador } \\
\text { retriever }\end{array}$ & $\begin{array}{l}\text { Ascites, pericardial } \\
\text { effusion }\end{array}$ & $\begin{array}{l}\text { III/VI holosystolic } \\
\text { bilateral apical }\end{array}$ & $\begin{array}{c}\text { Severe TVD + mild } \\
\text { MVD }\end{array}$ & $\begin{array}{c}\text { Euthanasia. Unresponsive } \\
\text { CHF }\end{array}$ \\
\hline $\operatorname{Dog} 22$ & $\begin{array}{l}\text { Springer } \\
\text { spaniel }\end{array}$ & $\begin{array}{l}\text { Heart murmur, } \\
\text { pulmonary oedema }\end{array}$ & $\begin{array}{l}\text { III/VI pansystolic } \\
\text { left apical }\end{array}$ & $\begin{array}{l}\text { Severe TVD + } \\
\text { Severe MVD }\end{array}$ & $\begin{array}{c}\text { Euthanasia. Unresponsive } \\
\text { CHF (left) }\end{array}$ \\
\hline $\operatorname{Dog} 23$ & Bullmastiff & $\begin{array}{l}\text { Arrhythmia, ascites, } \\
\text { pulmonary oedema }\end{array}$ & $\begin{array}{l}\text { III/VI systolic } \\
\text { bilateral apical }\end{array}$ & $\begin{array}{l}\text { Severe TVD + } \\
\text { Severe MVD }\end{array}$ & $\begin{array}{c}\text { Euthanasia. Unresponsive } \\
\text { CHF (bilateral) }\end{array}$ \\
\hline $\operatorname{Dog} 24$ & $\begin{array}{l}\text { Rottweiler } \\
\text { cross }\end{array}$ & $\begin{array}{l}\text { Arrhythmia, firm } \\
\text { head swelling }\end{array}$ & $\begin{array}{l}\text { VI/VI continuous } \\
\text { left basilar }\end{array}$ & $\begin{array}{c}\text { Severe TVD + PDA } \\
\text { (LtoR) }\end{array}$ & $\begin{array}{c}\text { Euthanasia. Unresponsive } \\
\text { seizures. }\end{array}$ \\
\hline $\operatorname{Dog} 25$ & $\begin{array}{l}\text { Labrador } \\
\text { retriever }\end{array}$ & Heart murmur & $\begin{array}{l}\text { III/VI pansystolic } \\
\text { right apical }\end{array}$ & $\begin{array}{c}\text { Mild TVD + small } \\
\text { ASD }\end{array}$ & $\begin{array}{l}\text { Euthanasia. Unresponsive } \\
\text { seizures. Suspected } \\
\text { meningioma }\end{array}$ \\
\hline $\operatorname{Dog} 26$ & $\begin{array}{l}\text { Border } \\
\text { collie }\end{array}$ & Heart murmur & $\begin{array}{l}\text { V/VI pansystolic } \\
\text { right apical }\end{array}$ & $\begin{array}{l}\text { Severe TVD + } \\
\text { small ASD }\end{array}$ & Alive \\
\hline $\operatorname{Dog} 27$ & $\begin{array}{l}\text { German } \\
\text { shepherd }\end{array}$ & Heart murmur & $\begin{array}{l}\text { VI/VI pansystolic } \\
\text { right apical }\end{array}$ & $\begin{array}{l}\text { Severe TVD + } \\
\text { severe PS }\end{array}$ & $\begin{array}{l}\text { Euthanasia. Meningeal } \\
\text { tumour. }\end{array}$ \\
\hline $\operatorname{Dog} 28$ & Boxer & Ascites, arrhythmia & $\begin{array}{c}\text { II/VI systolic right } \\
\text { apical }\end{array}$ & $\begin{array}{c}\text { Severe TVD + } \\
\text { moderate PS }\end{array}$ & $\begin{array}{c}\text { Natural. Cardiorespiratory } \\
\text { arrest }\end{array}$ \\
\hline $\operatorname{Dog} 29$ & $\begin{array}{l}\text { English } \\
\text { bulldog }\end{array}$ & $\begin{array}{l}\text { Heart murmur, } \\
\text { ascites, arrhythmia, } \\
\text { pleural and } \\
\text { pericardial effusion }\end{array}$ & $\begin{array}{l}\text { III/VI holosystolic } \\
\text { right apical }\end{array}$ & $\begin{array}{l}\text { Severe TVD + } \\
\text { severe PS }\end{array}$ & $\begin{array}{c}\text { Natural. Cardiorespiratory } \\
\text { arrest }\end{array}$ \\
\hline
\end{tabular}

(ASD): Atrial septal defect; (TVD): Tricuspid valve dysplasia; (SAS): Subaortic stenosis; (PS): Pulmonic stenosis; (MVD): Mitral valve dysplasia; (PDA): Patent ductus arteriosus; (LtoR): Left to right; (LTFU): Lost to follow up.

*Excluded from the descriptive and statistical analysis.

During initial clinical assessment, a systolic murmur was auscultated in all dogs, and ranged from grade I to VI out of VI (Table 2). In 16 dogs (89\%) the murmur was louder over the right thorax, while in two dogs $(11 \%)$, it had the same intensity bilaterally. The location of maximal intensity was right apical in 14 dogs $(78 \%)$ and right basilar in four dogs $(22 \%)$.

Standard electrocardiography was performed at least once in 14 dogs (78\%). Configuration abnormalities were identified in nine dogs $(50 \%)$ and consisted of a right axis shift $(\mathrm{n}=5,28 \%), \mathrm{P}$ pulmonale $(\mathrm{n}=4,22 \%)$ and splintered QRS $(\mathrm{n}=3,17 \%)$. Cardiac arrhythmias were reported at some point during the course of the disease in a total of four dogs $(22 \%)$. Atrial fibrillation was recorded in three dogs $(17 \%)$ and paroxysmal supraventricular tachycardia in 1/18 dogs (border collie).

Echocardiography identified thickened $(\mathrm{n}=16,89 \%)$, shortened $(n=4,22 \%)$ and elongated $(n=2,11 \%)$ TV leaflets. The septal leaflet appeared tethered in five dogs $(28 \%)$. Tricuspid stenosis was not observed in any dog. Other abnormalities of the TV apparatus included elongated PM $(n=1)$ and shortened CT $(n=1)$. TR was graded as severe in $11 \mathrm{dogs}(61 \%)$, moderate in two dogs $(11 \%)$ and mild in five dogs $(28 \%)$. The RA was severely enlarged in 11 dogs $(61 \%)$ and moderately enlarged in two dogs (11\%). RV enlargement was severe in four dogs $(22 \%)$, moderate in six dogs $(33 \%)$ and mild in two dogs $(11 \%)$. Chamber dimensions were within reference ranges in five dogs $(28 \%)$. Overall, TVD was graded as mild $(n=5,28 \%)$, moderate $(n=2$, $11 \%)$ or severe $(n=11,61 \%)$. EM was suspected in four dogs $(22 \%)$ based on the subjective apical displacement of the TV apparatus, but post-mortem diagnosis was not undertaken.

No association was observed between TVD severity and murmur intensity $(\mathrm{p}=0.198)$ or presence of an arrhythmia $(\mathrm{p}=0.371) \quad$ (Fig. 3); all dogs with arrhythmias had severe TVD.

Age at diagnosis of R-CHF ranged from 61 days to 7.8 years (median $=361$ days). Three $\operatorname{dogs}(17 \%)$ were more than one year old. Seven dogs were diagnosed with $\mathrm{R}-\mathrm{CHF}$ either on first presentation $(\mathrm{n}=4,22 \%)$ or subsequently $(n=3,17 \%)$, and all of them were diagnosed initially with severe TVD (Table 2). When R-CHF occurred, ascites was always present, pleural effusion (modified transudate) occurred in two dogs (11\%) and jugular distension/pulsation was reported in only one dog out of $18(6 \%)$. Thoracocentesis was required in one dog with pleural effusion to alleviate severe dyspnoea (modified transudate).

At the time of analysis, 12 out of 18 dogs (67\%) had died, four dogs were still alive $(22 \%)$ and two dogs (11\%) were LTFU. 


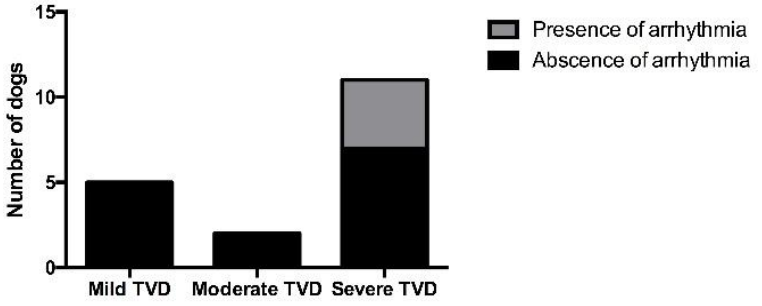

Fig. 3. Severity of TVD and presence of arrhythmias.

The seven dogs (39\%) diagnosed with R-CHF suffered cardiac death: six of them were euthanased (Table 2) due to unresponsive R-CHF, and one dog, with severe pleural effusion, died suddenly during thoracocentesis. Age at death ranged between 50 days and 13.8 years (median=2237.5 days). The MST from diagnosis of TVD (censored dogs $n=11,61 \%$ ) was 2775 days (range 1-3696 days; 95\% CI 1542.41-4007.59). However, once R-CHF developed (censored dogs $n=0$ ), MST was 181 days (range 1-2130 days; 95\% CI 0-455.59) (Fig. $4)$. None of the dogs without R-CHF suffered a cardiacrelated death. Dogs that suffered syncope were more likely to have shorter survival times (Hazard ratio $=21.814 ;$ 95\% CI 2.213-215.008, $\mathrm{p}=0.008$ ) (Fig. 4). The rest of the factors analysed, including age, sex, breed, TVD severity, HM intensity, R-CHF (at any point) and arrhythmia, were not significantly associated with survival time $(\mathrm{p}>0.05)$.

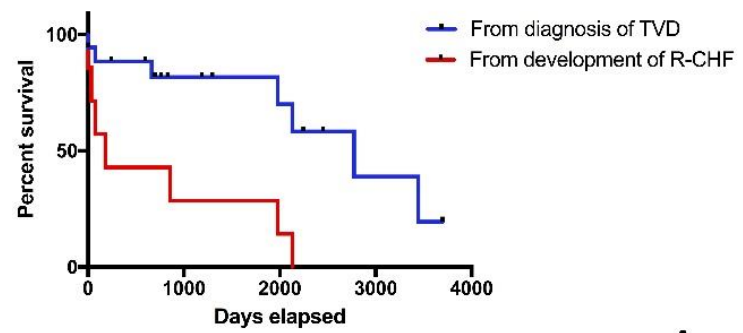

A

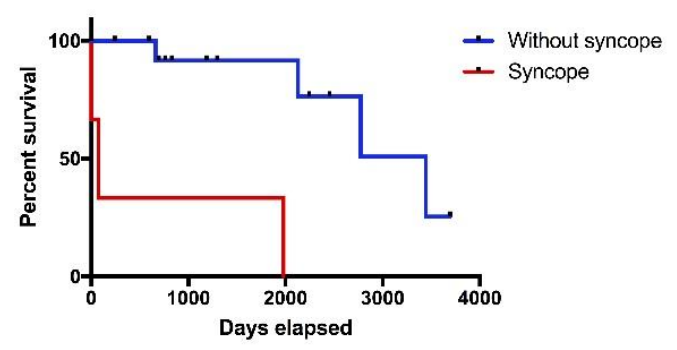

B

Fig. 4. Kaplan-Meier survival curves. (A): From diagnosis of TVD $(n=7)$ and from diagnosis of R-CHF $(n=7)$. (B): Dogs with $(n=3)$ and without $(n=4)$ syncope.

\section{Discussion}

This is the first study to document the demographic, clinical and survival characteristics of a UK population of dogs diagnosed with TVD. The prevalence was lower (1.1\% of our cardiology cases) than the $2-7.4 \%$ of congenital heart disease reported previously in nonUK populations (Tidholm, 1997; Oliveira et al., 2011; Schrope, 2015), but our reference population included both congenital and acquired cardiac disease. In addition, the true prevalence in the general UK population of dogs may be lower, as we are reporting from a referral population. Previous studies have suggested that Labrador retrievers are over-represented (Wright et al., 2001). In contrast, in our study, Labradors were not over-represented compared with the hospital population, but Border collies were significantly over-represented.

To our knowledge, a predisposition to TVD in Border collies has not been previously reported. Additional studies attempting to identify a mode of inheritance and potential genetic locus are warranted. More than one third of cases in this study had concomitant congenital cardiac diseases. This is consistent with previous studies in non-UK dogs (Oliveira et al., 2011; Adin, 2014) and people (Formigari et al., 1993; Edwards, 1994; Webb et al., 2008; Luu et al., 2015). There was also broad agreement with the types of congenital diseases previously reported, suggesting that TV development may be adversely affected during embryonic development if loading conditions in the heart are abnormal (Formigari et al., 1993). Although dogs with additional cardiac abnormalities were excluded from statistical analysis to minimise confounding effects, this study still demonstrates the importance of performing a comprehensive echocardiographic study, when TVD is diagnosed, to ensure additional congenital cardiac disease is not missed. Unsurprisingly for a congenital disease, the majority of TVD-only dogs were less than one year of age at diagnosis. However, one third of the dogs were adults. Diagnosing TVD in adult dogs can be controversial, as differentiating it from acquired chronic valvular disease can be difficult. Furthermore, degenerative valvular changes can also be observed in young dogs (Michaëlson and Ho, 2000). It is not clear as to why TVD was not diagnosed in so many dogs until adulthood, although this has been previously reported (Kittleson, 1998). Since inclusion criteria included the detection of a heart murmur before 1 year of age, it is possible that these dogs were not referred until the appearance of clinical signs or until the heart murmur intensity had increased. Only including dogs with a detectable heart murmur before of one year of age, may have precluded the inclusion of dogs with TVD but very low or undetectable heart murmurs, which is recognised in TVD cases (Famula et al., 2002). These 
dogs could even have had severe TVD since our study failed to demonstrate a correlation between murmur intensity and severity of TVD, as reported by previous authors (Bonagura and Lehmkuhl, 1999). Nonarrhythmic electrocardiographic abnormalities were relatively common. Although $P$ mitrale was not observed and splintering of the QRS complex was observed less frequently than in other studies (Kornreich and Moïse, 1997), P pulmonale and right axial shifts occurred at frequencies similar to those previously reported despite none of the dogs having precordial recordings (Kornreich and Moïse, 1997).

Cardiac arrhythmias were not frequently reported in our dogs. Neither ventricular pre-excitation nor the SVT typical of a macro re-entrant circuit was identified, despite an association between EM and WolffParkinson-White syndrome in people (Misaki et al., 1995), and the predisposition of Labradors to orthodromic atrioventricular reciprocating tachycardia (OAVRT) (Santilli et al., 2007). The only case in this study with SVT was a border collie, a breed not considered to be of increased risk for OAVRT. However, features of OAVRT may be intermittent, and since no Holter monitoring or electrophysiological studies were performed, concurrent OAVRT could not be completely ruled out. The most common arrhythmia was atrial fibrillation which is unsurprising since right atrial enlargement is a feature of severe TVD. Indeed, all dogs with cardiac arrhythmias had severe TVD. Surprisingly, a relationship between TVD severity and presence of an arrhythmia could not be demonstrated although the low number of events may have underpowered this investigation. We conclude that while cardiac remodelling is important in arrhythmogenesis in TVD, other factors, as yet unidentified, may also contribute to the arrhythmia risk. Doppler echocardiography was used to confirm the diagnosis and severity of TVD. TVD constitutes a wide range of morphological abnormalities (Boon, 2011b; Adin, 2014; Bussadori and Pradelli, 2016). The accurate and objective echocardiographic examination of the TV apparatus is technically challenging, and is not always feasible (Boon, 2011a). For example, the relatively thick and nodular appearance of the septal leaflet of the TV, irrespective of age, can mimic degenerative valvular disease (Michaëlson and Ho, 2000). Echocardiography cine loops were carefully rereviewed and diagnosis of congenital disease was subjectively achieved, based on typical morphological changes previously discussed (Table 1). Whether or not tethering of the septal tricuspid leaflet constitutes TVD is controversial. Although described in veterinary and human literature (Lang et al., 1991; Said et al., 2012) as resulting from an embryonic failure of programmed cell degeneration and inadequate undermining of the leaflet (Netter, 1981), other authors believe it to be a normal feature in dogs (Michaëlson and Ho, 2000). When we observed tethering to be preventing septal leaflet closure and in some occasions buckling away of the middle portion of the leaflet from the septum, it was considered part of TVD. This occurred in one third of our cases, although was not confirmed post-mortem. Similarly, a lack of post-mortem and/or electrophysiological data to demonstrate atrialisation of the RV, meant that EM, an unusual form of TVD (Eyster et al., 1977; Tilley and Liu, 1977; Cave, 2001; Takemura et al., 2003; Sousa et al., 2006; Choi et al., 2009), could not be confirmed in the four cases in which it was suspected. In agreement with Michaëlson and Ho (2000), we believe that a clearer distinction between EM and isolated TVD is required, and so these dogs were included as TVD-only dogs in statistical analysis. Overall, TVD had a favourable prognosis, with a MST, from diagnosis of TVD, of over 7 years. None of the mild-to-moderate cases (approximately two thirds of cases) suffered cardiac death. Only those cases diagnosed with severe TVD developed R-CHF, in the form of ascites, jugular distension/pulsation or pleural effusion. R-CHF occurred over a wide age range, in senior adults as well as juveniles, demonstrating that, as in humans and non-UK dogs, severe TVD can be tolerated for many years (Arizmendi et al., 2004; Webb et al., 2008; Oyama et al., 2010). Once R-CHF had developed, the MST was markedly reduced overall, although there was marked variation from a few days to over 5 years. Prolonged survival with TVD may reflect the capacity of the systemic venous vasculature to accommodate significant volume overload (MacDonald, 2006). Based on a small number of dogs reaching the endpoint, syncope was the sole risk factor for reduced survival time. Syncope only occurred in young dogs with severe TVD and R-CHF but the mechanism could not be identified from the data available. The only arrhythmia recorded in the syncopal dogs was paroxysmal SVT which would not normally lead to loss of consciousness (Moïse, 1999). However, it is possible that profound weakness due to inadequate cardiac output with or without an arrhythmia may have been misinterpreted as syncope. There were limitations to this study, mainly due to its retrospective nature, which may have precluded homogeneity of the data, and its dependency on accuracy of record keeping. To minimise inclusion of dogs with acquired disease, diagnosis of TVD in adult dogs also required historical identification of a right-sided murmur at a young age. It is still possible that dogs with acquired TV degeneration were erroneously included. Furthermore, there may have been a selection bias against dogs with TVD in whom a low intensity murmur had not been noted before one year of age. Review of stored echocardiographic images was not performed in a 
blinded manner, which may have biased classification of severity of TVD. Tricuspid regurgitation severity was assessed by evaluation of the regurgitant jet size. Colour-flow Doppler mapping is a simple, highly sensitive, semi-quantitative method of assessment of TR severity, with good repeatability and reproducibility, used very commonly in both human and veterinary medicine (Jacob and Stewart, 2007; Lancellotti et al., 2010; Boon, 2011a; Badano et al., 2013). However, it can be significantly affected by technical (transducer frequency, gain, frame rate, depth and Nyquist settings) and loading conditions (Jacob and Stewart, 2007; Lancellotti et al., 2010; Badano et al., 2013). Although studies in people suggest an acceptable correlation with TR severity (Zoghbi et al., 2003; Badano et al., 2013), currently, vena contracta width (VC), PISA radius and effective regurgitant orifice area (EROA), early diastolic inflow wave velocity (Evel) and hepatic vein flow, are considered superior quantitative markers of TR severity in humans (Lancellotti et al., 2010; Badano et al., 2013), and avoidance of jet area as a marker of severity has been recommended (Lancellotti et al., 2010).

To the authors' knowledge, there are no published studies evaluating the accuracy of different echocardiographic methods to assess TR severity in dogs. Recently, Sargent et al. (2015) reported a poor correlation of mitral regurgitant jet area and mitral regurgitation severity, in dogs with myxomatous mitral valve disease (MMVD). The same study identified Evel and VC indexed to aortic diameter (VC:Ao) as having the tightest correlations with mitral regurgitant fraction determined by cardiac magnetic resonance imaging, but whether indices of MR in MMVD could be applied to canine TVD is not known. Unfortunately, because our retrospective study used cases dating as far back as 1998, the cine loops necessary to calculate Evel and VC:Ao were not always available. In our study, and in order to maintain continuity and permit comparison with published studies of canine TVD (Ohad et al., 2013), we opted to use a combination of methods of assessing TVD severity, including TV apparatus abnormalities, severity of the right heart volume overload and, lastly, severity of TR.

In conclusion, TVD in our UK population of dogs was associated with a prolonged MST, from diagnosis of the disease, of over 7 years. R-CHF occurred only when TVD was severe. Once R-CHF developed, MST decreased but syncope was the sole risk factor for cardiac death. Border collies were over-represented.

\section{Acknowledgments}

The author would like to show his gratitude to Prof. Joanna Dukes-McEwan BVMS (Hons) MVM PhD DVC DipECVIM-CA(Cardiology) MRCVS, for her assistance and constructive criticism of the manuscript.

\section{Conflict of interest}

The authors declare that there is no conflict of interest.

\section{References}

Adams, D.S., Marolf, A.J., Valdés-Martinez, A., Randall, E.K. and Bachand, A.M. 2017. Associations between thoracic radiographic changes and severity of pulmonary arterial hypertension diagnosed in 60 dogs via Doppler echocardiography: A retrospective study. Vet. Radiol. Ultrasound. 58, 454-462.

Adin, D. 2014. Tricuspid valve dysplasia. In Kirk's Current Veterinary Therapy, Eds., Bonagura, J.D. and Twedt, D.C. Saint Louis, MI: W.B. Saunders, pp: e332-e335.

Andelfinger, G., Wright, K.N., Lee, H.S., Siemens, L.M. and Benson, D.W. 2003. Canine tricuspid valve malformation, a model of human Ebstein anomaly, maps to dog chromosome 9. J. Med. Genet. 40, 320-324.

Arizmendi, A.F., Pineda, L.F., Jiménez, C.Q., Azcárate, M.J.M., Sarachaga, I.H., Urroz, E., de León, J.P., Moya, J.L. and Jiménez, M.Q. 2004. The clinical profile of Ebstein's malformation as seen from the fetus to the adult in 52 patients. Cardiol. Young. 14, 55-63.

Badano, L.P., Muraru, D. and Enriquez-Sarano, M. 2013. Assessment of functional tricuspid regurgitation. Eur. Heart J. 34, 1875-1884.

Becker, A.D., Becker, M.J. and Edwards, J.E. 1971. Pathologic spectrum of dysplasia of the tricuspid valve: features in common with Ebstein's malformation. Arch. Pathol. 91, 167-178.

Bonagura, J.D. and Lehmkuhl, L.B. 1999. Congenital heart disease. In Textbook of Canine and Feline Cardiology: Principles and Clinical Practice, Eds., Fox, P.R., Sisson, D. and Moïse, N.S. Philadelphia, PA: W.B. Saunders, pp: 471-535.

Boon, J.A. 2011a. Evaluation of size, function and haemodynamics. In Veterinary Echocardiography, Eds., Boon, J.A. Ames, IA: John Willey \& Sons, pp: 153-266.

Boon, J.A. 2011b. Congenital shunts and AV dysplasia. In Veterinary Echocardiography, Eds., Boon, J.A. Ames, IA: John Willey \& Sons, pp: 437-475.

Boon, J.A. 2011c. Acquired valvular disease. In Veterinary Echocardiography, Eds., Boon, J.A. Ames, IA: John Willey \& Sons, pp: 267-333.

Bussadori, C. and Pradelli, D. 2016. Congenital cardiopathies. In Clinical Echocardiography of the Dog and Cat, Eds., Chetboul, V., Bussadori, C. and de Madron, E. St. Louis, MI: Elsevier, pp: 283-322.

Cave, T. 2001. What is your diagnosis? Congenital tricuspid dysplasia with concurrent Ebstein's anomaly. J. Small Anim. Pract. 42, 311-314. 
Choi, R., Lee, S.K., Moon, H.S., Park, I.C. and Hyun, C. 2009. Ebstein's anomaly with an atrial septal defect in a jindo dog. Can. Vet. J. 50, 405-410.

Edwards, W.D. 1994. Embryology and pathologic features of Ebstein's anomaly. Progr. Pediatr. Cardiol. 2, 5-15.

Eyster, G.E., Anderson, L., Evans, A.T., Chaffee, A., Bender, G., Johnston, J., Muir, W. and Blanchard, G. 1977. Ebstein's anomaly: a report of 3 cases in the dog. J. Am. Vet. Med. Assoc. 170, 709-713.

Famula, T.R., Siemens, L.M., Davidson, A.P. and Packard, M. 2002. Evaluation of the genetic basis of tricuspid valve dysplasia in Labrador Retrievers. Am. J. Vet. Res. 63, 816-820.

Formigari, R., Francalanci, P., Gallo, P., D’Offizi, F., di Gioia, C., Hokayern, N.J., D'Alessandro, C. and Colloridi, V. 1993. Pathology of atrioventricular valve dysplasia. Cardiovasc. Pathol. 2, 137-144.

Jacob, R. and Stewart, W.J. 2007. A practical approach to the quantification of valvular regurgitation. Curr. Cardiol. Rep. 9, 105-111.

Kittleson, M.D. 1998. Congenital abnormalities of the atrioventricular valves. In Small Animal Cardiovascular Medicine, Eds., Kittleson M.D. and Kienle R.D. St. Louis, MI: Mosby, pp: 273-281.

Kornreich, B.G. and Moïse, N.S. 1997. Right atrioventricular valve malformation in dogs and cats: An electrocardiographic survey with emphasis on splintered QRS complexes. J. Vet. Intern. Med. 11, 226-230.

Lancellotti, P., Moura, L., Pierard, L.A., Agricola, E., Popescu, B.A., Tribouilloy, C., Hagendorff, A., Monin, J.L., Badano, L. and Zamorano, J.L. 2010. European Association of Echocardiography recommendations for the assessment of valvular regurgitation. Part 2: mitral and tricuspid regurgitation (native valve disease). Eur. J. Echocardiogr. 11, 307-332.

Lake-Bakaar, G.A., Griffiths, L.G. and Kittleson, M.D. 2017. Balloon Valvuloplasty of Tricuspid Stenosis: A Retrospective Study of 5 Labrador Retriever Dogs. J. Vet. Intern. Med. 31, 311-315.

Lang, D., Oberhoffer, R., Cook, A., Sharland, G., Allan, L., Fagg, N. and Anderson, R.H. 1991. Pathologic spectrum of malformations of the tricuspid valve in prenatal and neonatal life. J. Am. Coll. Cardiol. 17, 1161-1167.

Lang, R.M., Bierig, M., Devereux, R.B., Flachskampf, F.A., Foster, E., Pellikka, P.A., Picard, M.H., Roman, M.J., Seward, J., Shanewise, J., Solomon, S., Spencer, K.T., St John Sutton, M., Stewart, W.; American Society of Echocardiography's Nomenclature and Standards Committee; Task Force on Chamber Quantification; American College of Cardiology Echocardiography Committee; American Heart Association; European
Association of Echocardiography, European Society of Cardiology. 2006. Recommendations for chamber quantification. Eur. J. Echocardiogr. 7(2), 79-108.

Luu, Q., Choudhary, P., Jackson, D., Canniffe, C., McGuire, M., Chard, R. and Celermajer, D.S. 2015. Ebstein's anomaly in those surviving to adult life a single centre experience. Heart Lung Circ. 24, 996-1001.

MacDonald, K.A. 2006. Congenital heart diseases of puppies and kittens. Vet. Clin. North Am. Small Pract. 36, 503-531.

Michaëlson, M. and Ho, S.Y. 2000. Comparative aspects. In Congenital Heart Malformations in Mammals, Eds., Michaëlson, M. and Ho, S.Y. London: Imperial College Press, pp: 119-150.

Misaki, T., Watanabe, G., Iwa, T., Watanabe, Y., Mukai, K., Takahashi, M., Ohtake, H. and Yamamoto, K. 1995. Surgical treatment of patients with Wolff-Parkinson-White syndrome and associated Ebstein's anomaly. J. Thorac. Cardiovasc. Surg. 110, 1701-1707.

Moïse, N. S. 1999. Diagnosis and management of canine arrhythmias. In Textbook of Canine and Feline Cardiology: Principles and Clinical Practice, Eds., Fox, P.R., Sisson, D. and Moïse, N.S. Philadelphia, PA: W.B. Saunders, pp: 331-385.

Netter, F.H. 1981. The CIBA Collection of Medical Illustration. Vol 5: Heart. Ciba-Geigy Corporation.

Ohad, D.G., Avrahami, A., Waner, T. and David, L. 2013. The occurrence and suspected mode of inheritance of congenital subaortic stenosis and tricuspid valve dysplasia in Dogue de Bordeaux dogs. Vet. J. 197, 351-357.

Oyama, M.A., Sisson, D.D., Thomas, W.P. and Bonagura, J.D. 2010. Congenital heart disease. In Textbook of Veterinary Internal Medicine Vol. 2, Eds., Ettinger, S.J. and Feldman, E.C. St. Louis, MI: W.B. Saunders, pp: 1251-1298.

Oliveira, P., Domenech, O., Silva, J., Vannini, S., Bussadori, R. and Bussadori, C. 2011. Retrospective review of congenital heart disease in 976 dogs. J. Vet. Intern. Med. 25, 477-483.

Said, S.M., Burkhart, H.M. and Dearani, J.A. 2012. Surgical management of congenital (Non-Ebstein) tricuspid valve regurgitation. Semin. Thorac. Cardiovasc. Surg. Pediatr. Card. Surg. Annu. 15, 46-60.

Santilli, R.A., Spadacini, G., Moretti, P., Perego, M., Perini, A., Crosara, S. and Tarducci, A. 2007. Anatomic distribution and electrophysiologic properties of accessory pathways in dogs. J. Am. Vet. Med. Assoc. 231, 393-398.

Sargent, J., Connolly, D.J., Watts, V., Motsküla, P., Volk, H.A., Lamb, C.R. and Fuentes, V.L. 2015. Assessment of mitral regurgitation in dogs: 
comparison of results of echocardiography with magnetic resonance imaging. J. Small Anim. Pract. 56, 641-650.

Schrope, D.P. 2015. Prevalence of congenital heart disease in 76,301 mixed-breed dogs and 57,025 mixed-breed cats. J. Vet. Cardiol. 17, 192-202.

Shiina, A., Seward, J.B. and Edwards, W.D. 1984. Two-dimensional echocardiographic spectrum of Ebstein's anomaly: detailed anatomic assessment. J. Am. Coll. Cardiol. 3, 356-357.

Sousa, M.G., Gerardi, D.G., Alves, R.O. and Camacho, A.A. 2006. Tricuspid valve dysplasia and Ebstein's anomaly in dogs: case report. Arq. Bras. Med. Vet. Zootec. 58, 762-767.

Takemura, N., Machida, N., Nakagawa, K., Amasaki, H., Washizu, M. and Hirose, H. 2003. Ebstein's anomaly in a beagle dog. J. Vet. Med. Sci. 65, 531533.

Tidholm, A. 1997. Retrospective study of congenital heart defects in 151 dogs. J. Small Anim. Pract. 38, 94-98.

Tilley, L.P. and Liu, S.K. 1977. Ebstein's anomaly. J.
Am. Med. Assoc. 171, 798-802.

Webb, G.D., Smallhorn, J.F., Therrien, J. and Redington, A.N. 2008. Disease of the heart, pericardium, and pulmonary vasculature bed. In Braunwald's Heart Disease: A Textbook of Cardiovascular Medicine, Eds., Libby, P., Bonow, R.O., Mann, D.L., Zipes, D.P., Braunwald, E. St. Louis, MI: W.B. Saunders, pp: 1561-1625.

Wright, K.N., Bleas, M.E. and Benson, D.W. 2001. Clinical spectrum of congenital tricuspid valve malformation in an extended family of Labrador retrievers. J. Vet. Intern. Med. 15, 280.

Zoghbi, W.A., Enriquez-Sarano, M., Foster, E., Grayburn, P.A., Kraft, C.D., Levine, R.A., Nihoyannopoulos, P., Otto, C.M., Quinones, M.A., Rakowski, H., Stewart, W.J., Waggoner, A., Weissman, N.J.; American Society of Echocardiography. 2003. Recommendations for evaluation of the severity of native valvular regurgitation with two-dimensional and Doppler echocardiography. J. Am. Soc. Echocardiogr. 16, 777-802. 